\title{
CONE-BEAM COMPUTED TOMOGRAPHY EVALUATION OF THE RELATIONSHIP BETWEEN MAXILLARY TRANSVERSE DIMENSIONS AND MAXILLARY IMPACTED CANINES
}

\author{
Shaimaa Mohammed Elmarhoumy*
}

\begin{abstract}
Aim: Evaluate the relationship between maxillary transverse dimensions and impacted maxillary canines using cone beam computed tomography (CBCT).

Materials and Methods: This retrospective radiographic study reviewed 45 CBCT records of 45 subjects their ages above 12 years. The records were divided into 3 groups, Group I: normally erupted maxillary canine group (control group), group II: labial impacted maxillary canine group, group III: palatal impacted maxillary canine group. Maxillary width was measured at 4 levels: basal first molar width, alveolar first molar width, basal first premolar width, and alveolar first premolar width. The depth of the palatal vault was measured to evaluate the shape of the palate in all groups.

Results: labial and palatal impacted groups showed significantly smaller basal first molar widths, alveolar first molar and first premolar widths, compared with the control group $(\mathrm{P}<0.05)$. Basal first premolar widths and palatal vault depths were not significantly different between the groups.
\end{abstract}

Conclusions: Maxillary width was smaller in subjects with labial or palatal maxillary canine impaction than subjects without impaction.

KEYWORDS: Impacted canines; Maxillary transverse discrepancy; CBCT

\section{INTRODUCTION}

Maxillary permanent canine is considered the foremost tooth within the dental arch that plays a vital role in smile design. Any eruption problem affecting the maxillary permanent canine incorporates a negative effect on the smile and facial appearance.
The maxillary permanent canine is one of the most predominant teeth to be impacted second to the third molars ${ }^{1}$, with a prevalence of impaction ranges from $1-3 \%$ of the population ${ }^{2}$. Palatal canine impaction occurs 3 to 6 times more than buccal impaction ${ }^{3}$.

Traditionally, the position of impacted canines

\footnotetext{
* Lecturer of Orthodontics, Faculty of Dentistry, Tanta University, Egypt.
} 
has been determined by periapical and/or panoramic radiographs. However, these radiographs are a twodimensional image of a three-dimensional object. Additionally, panoramic radiograph has other disadvantages like, image distortion and overlapping of adjacent structures to impacted canine resulting in difficulty in assessing impacted canine labiopalatally ${ }^{4}$. Recently, cone beam computed tomography (CBCT) can be utilized to locate impacted teeth because it determines the three-dimensional position of the impacted canine accurately and estimate root resorption of the adjacent teeth efficiently ${ }^{5}$.

Various studies evaluated the relationship between the morphology of the maxilla and the impaction of maxillary canines, but their results often were contradictory. McConell et al., ${ }^{6}$ compared dental casts of 57 subjects with palatally displaced canines to 103 subjects without canine impaction. They considered deficiency in maxillary transverse width as one of the causes of palatally impacted maxillary permanent canines.

Langberg and Peck ${ }^{7}$ examined pretreatment diagnostic casts of patients with palatally impacted maxillary canines and demonstrated no statistically significant difference within the anterior and posterior widths of the maxilla between palatally impacted canine group and the control group.

Sambatro et al., ${ }^{8}$ took posteroanterior cephalometric radiographs of 43 patients aged 8 years old, and again at 14.3 years for the same patients. They observed a wider posterior maxillary width for patients who later displayed impacted maxillary canines. Moreover, another study by Al-Nimri and Gharaibeh ${ }^{9}$ on pretreatment dental casts showed greater width of the maxillary arch in subjects with palatally displaced canines than those with normally erupted canines.

Saiar et al. ${ }^{10}$ studied the casts and posteroanterior cephalograms of 79 patients with palatally impacted canines and found no significant relation between the skeletal maxillary width and the palatally displaced canines. So, they concluded that deficiency in maxillary arch width could be considered as an effect rather than a cause of impaction. Thus, suggesting that no benefit might be gained by early expansion of the maxillary arch in patients with canine impaction.

Association between occurrence of palatally displaced canines and maxillary transverse discrepancy in the mixed dentition was studied by Schindel and Duffy ${ }^{11}$. They used casts and panoramic radiographs of 84 patients with transverse maxillary deficiency and 100 as a control. Sector classification on panoramic radiographs were used for prediction of canine impaction for both the groups. They revealed that transverse discrepancies of the maxillary arch increase the chance of canine impaction. In addition, Ali Rizvil et al., ${ }^{12}$ measured the maxillary arch width of 60 casts as the distance between the mesiopalatal cusp tips of the first permanent molars, and the mandibular arch width as the distance between the central fossae of the mandibular first permanent molars. Deficiency of maxillary width was calculated as the difference between the two measurements. They observed a positive correlation between transverse maxillary deficiency and occurrence of maxillary canine impaction. So, they concluded that, early treatment of transverse deficiency of the maxillary arch may decrease the probability of maxillary canine impaction.

However, Fattahi et al., ${ }^{13}$ and Refaat and ElDesouky ${ }^{14}$ demonstrated that, no statistically significant difference in inter molar width, inter premolar width, inter canine width, between the impaction groups (buccal and palatal impaction) and the control group. Furthermore, Kim et al., ${ }^{15}$ assessed the relationship between the position of maxillary impacted canines and the morphology of the maxilla using arch length / inter molar width $\mathrm{x} 100$ as index of maxillary arch shape and palatal vault depth / inter molar width $x 100$ as index of palatal shape. They concluded that patients with palatally impacted canine showed smaller maxillary width and deeper palatal vault compared with those of buccally impacted canine. 
Hong et al., ${ }^{5}$, Yan et al., ${ }^{16}$ and Gull et al., ${ }^{17}$ evaluated the pretreatment CBCT of subjects with palatally impacted canines and concluded that the skeletal and dental maxillary transverse widths on the first molar, second premolar, and first premolar level had no effect on the occurrence of palatally impacted canines. Additionally, a recent crosssectional study was conducted by Ghaffar et al., ${ }^{18}$ on 85 panoramic radiographs and dental casts of Pakistani patients. Deficiency in transverse maxillary dimension was calculated on dental cast by subtracting the mandibular intermolar width from the maxillary intermolar width. Prediction of maxillary impacted canines was done on panoramic radiographs based on sectors classification. They concluded that there was a higher risk of palatal impaction of maxillary canine in patients with narrower maxillary arch.

Ariza et al., ${ }^{19}$ compared 86 CBCT images of patients with maxillary impacted canines and 67 images of patients with normally erupted maxillary canine as a control. They found smaller maxillary widths at first molars and first premolars levels in impacted maxillary canine group compared with the control group. Also, Cacciatore et al., ${ }^{20}$ calculated seven measurements on the digital casts of 49 patients had a mean age of 9 years old to evaluate the relationship between early diagnosed impacted maxillary canines, using panoramic radiographs, and the morphology of the maxilla. They reported narrower and shorter maxillary arch in patients with displaced maxillary canines compared with subjects without eruption problem.

More recently, a retrospective CBCT study was conducted by Guillen et al., ${ }^{21}$ to assess the changes of maxillary width at premolar level after traction of impacted maxillary canines in young adults. They reported increase in the maxillary inter-premolar widths after traction of impacted maxillary canines until the occlusal plane.

Nonetheless, it still difficult to clarify the causes of impaction of maxillary canine. The relationship between the maxillary width, the palatal vault depth, and the position of impacted maxillary canine requires further investigation. Thus, the purpose of this study was to assess whether there was a relationship between the width of the maxilla, the depth of the palatal vault and the occurrence of impacted maxillary canines, using CBCT.

\section{MATERIAL AND METHODS}

The study sample consisted of 45 CBCT images of Egyptian patients with impacted maxillary canine, their ages above 12 years. CBCT images were collected from Orthodontic department, Faculty of Dentistry, Tanta University and private radiology centers.

The minimum number of sample size for this study was calculated and found to be 45 patients. The criteria used for sample size calculation were as follows: $95 \%$ confidence limit, $80 \%$ power of the study. The following equation was used for sample size calculation:

$$
\mathrm{N}=\frac{\left(Z_{\alpha}\right)^{2} *(S D)^{2}}{(d)^{2}}
$$

$\mathrm{N}=$ Total sample size, $Z_{\alpha}=$ Is standard normal variate and its equal 4.39 at $\mathrm{P}<0.05$

$S D=$ Standard deviation of variable

$d=$ Absolute error or precision

\begin{tabular}{|c|c|c|}
\hline$Z_{\alpha}$ & $S D$ & $d$ \\
\hline 4.39 & 3.05 & 2 \\
\hline
\end{tabular}

Total sample size $\mathrm{n}=\frac{(4.39)^{2} *(3.05)^{2}}{(2)^{2}}$
$=44.8 \approx 45$ samples.

Subjects with systemic disease, previous orthodontic treatment, craniofacial deformities, cleft lip or palate, head and neck syndromes, history of trauma, odontoma, supernumerary, several impacted or congenitally missing teeth were excluded. 
CBCT scan was acquired with a DCT Pro*. Scanning parameters was $90 \mathrm{kVp}, 24 \mathrm{~s}, 4 \mathrm{~mA}$, voxel size $0.4 \mathrm{~mm}$ and field of view $20 \times 19 \mathrm{~cm}$. Using CBCT data, the subjects were divided into 3 groups, group I: normally erupted maxillary canine group (control group), group II: labial impacted maxillary canine group, group III: palatal impacted maxillary canine group.

For maxillary width measurements, DICOM files were imported into Mimics software (version 10.0; Materialise, Leuven, Belgium) that was used to orient the CBCT scans and measure all data. Maxillary widths were measured at 4 levels: basal first molar width (BMW), alveolar first molar width (AMW), basal first premolar width (BPMW), and alveolar first premolar width (APMW) (Fig 1).
The lowest point on the right and left sides of the nasal floor were used to draw a nasal floor reference plane. First molar measurements were made on the most anterior coronal image showing the roots furcation. Premolar measurements were made on the coronal image showing the center of the root canal. Palatal vault depth was measured to assess the shape of the palate among groups. The definitions of the CBCT measurements are shown in Table I.

Measurements were repeated 2 weeks after the initial measurements on 24 randomly selected samples, 8 from each group. The reliability of these repeated measurements was tested using the intraclass correlation coefficient (ICC) with a $95 \%$ confidence interval. ICC values ranged from 0.91 to 0.96 , which indicated a high degree of reliability.
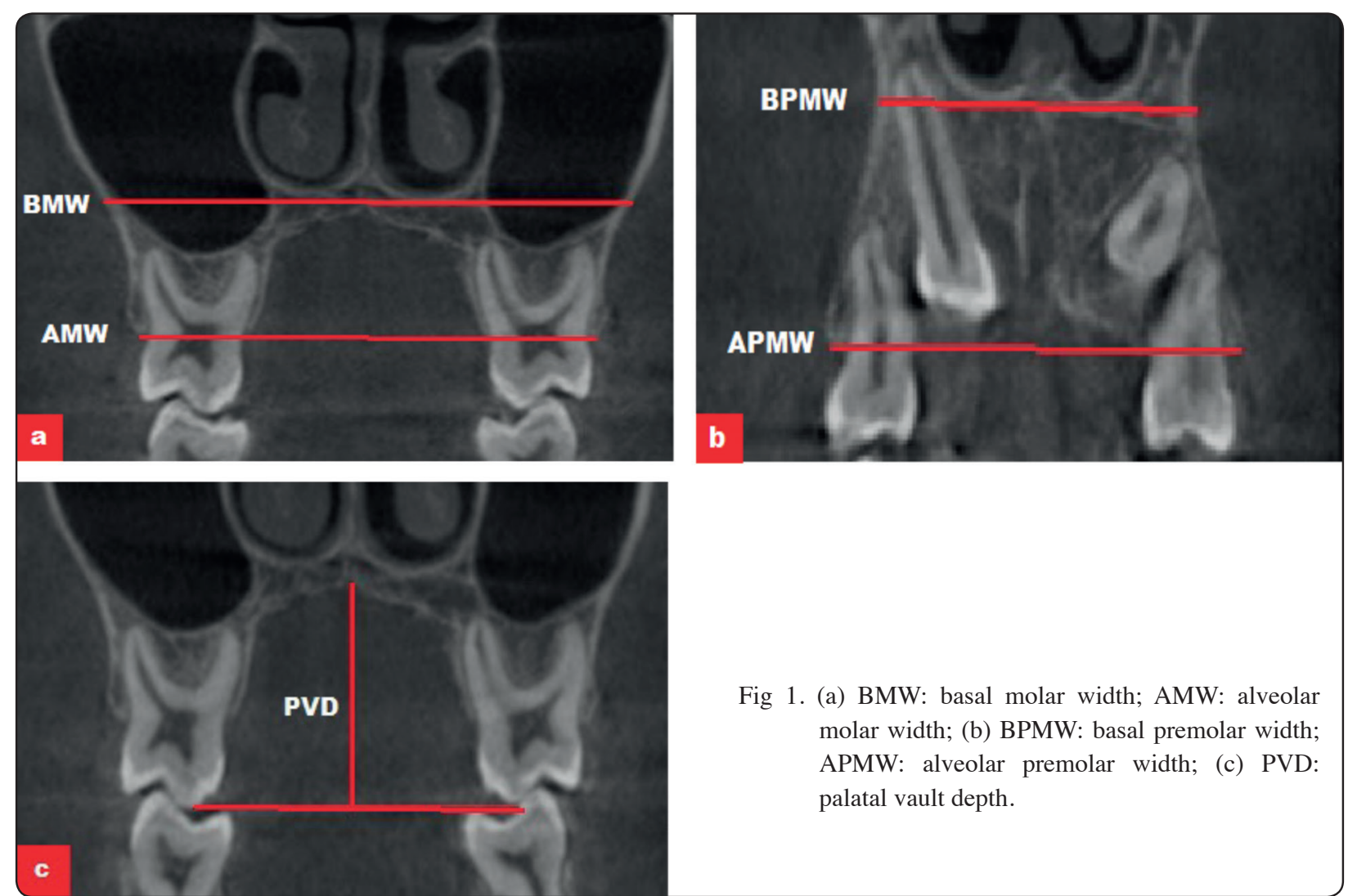

Fig 1. (a) BMW: basal molar width; AMW: alveolar molar width; (b) BPMW: basal premolar width; APMW: alveolar premolar width; (c) PVD: palatal vault depth.

* (Planmeca, Helsinki, Finland)

** (Vatech Co., Hwasung, Republic of Korea) 
TABLE (I) Definition of the variables used in this study according to Ariza et al., 19.

\begin{tabular}{|l|l|}
\hline Variable & Definition \\
\hline BMW & $\begin{array}{l}\text { The basal first molar width was measured between the most outer points of the right and left sides of the maxillary } \\
\text { base along the nasal floor reference plane. }\end{array}$ \\
\hline AMW & $\begin{array}{l}\text { The alveolar first molar width was measured between the most occlusal right and left points of the maxillary } \\
\text { alveolar process. }\end{array}$ \\
\hline BPMW & $\begin{array}{l}\text { The basal first premolar width was measured between the most outer points of the right and left sides of the } \\
\text { maxillary base along the nasal floor reference plane. }\end{array}$ \\
\hline APMW & $\begin{array}{l}\text { The alveolar first premolar width was measured between the most occlusal right and left points of the maxillary } \\
\text { alveolar process. }\end{array}$ \\
\hline PVD & $\begin{array}{l}\text { The palatal vault depth was measured between the deepest point of the palatal vault and the line connecting } \\
\text { between the mesio-palatal cusps tips of the right and left first molars. }\end{array}$ \\
\hline
\end{tabular}

\section{Statistical Analysis}

The data were collected, tabulated, and analyzed. Statistical analysis was performed with SPSS Version 21.0 (SPSS Inc, Chicago, III). Independent t-test was used for comparing the mean changes between the groups.

\section{RESULTS}

The average values and standard deviations for each parameter used for assessment of maxillary morphology in the different groups are compared in tables (2,3 and 4$)$.

Regarding maxillary width measurements, there were statistically significant greater BMW, AMW, and APMW in the control group compared with the labial and palatal impaction groups. There were no statistically significant differences between the two impaction groups (labial and palatal groups) for any parameters.

For the BMW measurements, there were significant differences of $3.11 \mathrm{~mm}$ between the control group $(66.14 \mathrm{~mm})$ and the labial group $(63.03 \mathrm{~mm}$ ) (Table 2). And $4.55 \mathrm{~mm}$ between the control and the palatal groups $(61.59 \mathrm{~mm})$ (Table 3$)$, $\mathrm{P}<0.05$. As regard to AMW measurements, there were significant differences of $3.11 \mathrm{~mm}$ between

TABLE (2): Comparison of maxillary transverse width measurements and palatal vault depth between control and labial groups

\begin{tabular}{|c|c|c|c|c|c|}
\hline & & Control & Labial & t. test & p. value \\
\hline \multirow{2}{*}{ BMW } & Range & $57.99-76.87$ & $55.56-67.49$ & \multirow{2}{*}{2.092} & \multirow{2}{*}{$0.045^{*}$} \\
\hline & Mean \pm S. D & $66.14 \pm 4.91$ & $63.03 \pm 3.10$ & & \\
\hline \multirow{2}{*}{ AMW } & Range & $54.7-64.05$ & $48.82-59.12$ & \multirow{2}{*}{2.623} & \multirow{2}{*}{$0.014^{*}$} \\
\hline & Mean \pm S. D & $58.08 \pm 3.06$ & $54.97 \pm 3.53$ & & \\
\hline \multirow{2}{*}{ BPMW } & Range & $32.37-42.85$ & $31.75-43.05$ & \multirow{2}{*}{1.992} & \multirow{2}{*}{0.056} \\
\hline & Mean \pm S. D & $37.49 \pm 3.22$ & $39.74 \pm 3.06$ & & \\
\hline \multirow{2}{*}{ APMW } & Range & $42.62-53.05$ & $39.32-47.77$ & \multirow{2}{*}{2.761} & \multirow{2}{*}{$0.010^{*}$} \\
\hline & Mean \pm S. D & $47.72 \pm 3.21$ & $44.90 \pm 2.38$ & & \\
\hline \multirow{2}{*}{ PD } & Range & $15.71-32.2$ & $18.07-24.55$ & \multirow{2}{*}{0.237} & \multirow{2}{*}{0.814} \\
\hline & Mean \pm S. D & $21.39 \pm 3.83$ & $21.12 \pm 2.08$ & & \\
\hline
\end{tabular}


the control group $(58.08 \mathrm{~mm}$ ) and the labial group (54.97 $\mathrm{mm}$ ) (Table 2), and $4.29 \mathrm{~mm}$ between the control and the palatal groups $(53.79 \mathrm{~mm})$ (Table $3), \mathrm{P}<0.05$. Concerning APMW measurements, there were also significant differences between the control group, the labial and palatal groups (2.82 and $3.8 \mathrm{~mm}$, respectively; $\mathrm{P}<0.05)$. No significant differences were recorded in the BPMW and PVD measurements among the 3 groups. Also, there were no statistically significant differences between labial and palatal groups regarding to all variables (Table 4).

TABLE (3): Comparison of maxillary transverse width measurements and palatal vault depth between control and palatal groups

\begin{tabular}{|c|c|c|c|c|c|}
\hline & & Control & Palatal & t. test & p. value \\
\hline \multirow{2}{*}{ BMW } & Range & $57.99-76.87$ & $54.8-68.18$ & \multirow{2}{*}{2.698} & \multirow{2}{*}{$0.011^{*}$} \\
\hline & Mean \pm S. D & $66.14 \pm 4.91$ & $61.59 \pm 4.45$ & & \\
\hline \multirow{2}{*}{ AMW } & Range & $54.7-64.05$ & $47.8-60.31$ & \multirow{2}{*}{3.354} & \multirow{2}{*}{$0.002 *$} \\
\hline & Mean \pm S. D & $58.08 \pm 3.06$ & $53.79 \pm 4.02$ & & \\
\hline \multirow{2}{*}{ BPMW } & Range & $32.37-42.85$ & $29.35-47.92$ & \multirow{2}{*}{1.300} & \multirow{2}{*}{0.204} \\
\hline & Mean \pm S. D & $37.49 \pm 3.22$ & $35.27 \pm 5.98$ & & \\
\hline \multirow{2}{*}{ APMW } & Range & $42.62-53.05$ & $38.51-49.57$ & \multirow{2}{*}{2.918} & \multirow{2}{*}{$0.007 *$} \\
\hline & Mean \pm S. D & $47.72 \pm 3.21$ & $43.92 \pm 4.03$ & & \\
\hline \multirow{2}{*}{ PD } & Range & $15.71-32.2$ & $15.13-24.42$ & \multirow{2}{*}{0.152} & \multirow{2}{*}{0.880} \\
\hline & Mean \pm S. D & $21.39 \pm 3.83$ & $21.57 \pm 2.79$ & & \\
\hline
\end{tabular}

TABLE (4) Comparison of maxillary transverse width measurements and palatal vault depth between labial and palatal groups.

\begin{tabular}{|c|c|c|c|c|c|}
\hline & & Labial & Palatal & t. test & p. value \\
\hline \multirow{2}{*}{ BMW } & Range & $55.56-67.49$ & $54.8-68.18$ & \multirow{2}{*}{1.031} & \multirow{2}{*}{0.311} \\
\hline & Mean \pm S. D & $63.03 \pm 3.10$ & $61.59 \pm 4.45$ & & \\
\hline \multirow{2}{*}{ AMW } & Range & $48.82-59.12$ & $47.8-60.31$ & \multirow{2}{*}{0.853} & \multirow{2}{*}{0.401} \\
\hline & Mean \pm S. D & $54.97 \pm 3.53$ & $53.79 \pm 4.02$ & & \\
\hline \multirow{2}{*}{ BPMW } & Range & $31.75-43.05$ & $29.35-47.92$ & \multirow{2}{*}{1.764} & \multirow{2}{*}{0.089} \\
\hline & Mean \pm S. D & $38.54 \pm 3.94$ & $35.27 \pm 5.98$ & & \\
\hline \multirow{2}{*}{ APMW } & Range & $39.32-47.77$ & $38.51-49.57$ & \multirow{2}{*}{0.817} & \multirow{2}{*}{0.421} \\
\hline & Mean \pm S. D & $44.90 \pm 2.38$ & $43.92 \pm 4.03$ & & \\
\hline \multirow{2}{*}{ PD } & Range & $18.07-24.55$ & $15.13-24.42$ & \multirow{2}{*}{0.502} & \multirow{2}{*}{0.620} \\
\hline & Mean \pm S. D & $21.12 \pm 2.08$ & $21.57 \pm 2.79$ & & \\
\hline
\end{tabular}




\section{DISCUSSION}

The aim of the present study was to compare the maxillary width and the palatal vault depth in subjects with labial and palatal impacted maxillary canines versus subjects with normal maxillary canine eruption as a control, using CBCT. Although many studies have been directed to assess the relationship between impacted maxillary canines and the morphology of the maxilla, their results are controversial and sometimes, contradictory ${ }^{5,7,11}$.

Only patients above 12 years of age were included in this study because radiographic examination before the age of 11 years does not allow an accurate prognosis for maxillary canine eruption path because the root of the maxillary canine begins to deflect buccally and occlusally at the age of 10 years ${ }^{22}$.

Cone beam computed tomography (CBCT) is considered the most accurate diagnostic tool to locate impacted teeth with lower exposure dose, lower cost, and better image accuracy than conventional CT. Also, it overcomes the blurring of image, overlapping of adjacent structures, and the superimpositions inherent in panoramic radiographs ${ }^{23,24}$.

The findings of the current study revealed significant smaller inter molar and inter premolar maxillary width in impacted maxillary canine groups (labial impaction and palatal impaction) compared with control group (Table 2-3). These results agreed with other studies ${ }^{6,11,12,20}$ that evaluated the maxillary width and morphology on study models and found that patients with impacted maxillary permanent canines had narrower maxilla than subjects without eruption problems.

Tadinada et al., ${ }^{25}$ and Aracena et al., ${ }^{26}$ demonstrated decrease in maxillary width on the impacted sides in subjects with unilateral palatally impacted canines using CBCT. Also, Ariza et al., ${ }^{19}$ measured the maxillary transverse dimensions utilizing CBCT and found a smaller maxillary width in impacted maxillary canine group compared with normally erupted maxillary canine group. Guillen et al., ${ }^{21}$ even stated that, traction of maxillary impacted canines increases the maxillary inter premolar widths (first or second) without differences between type (unilateral versus bilateral) or location (buccal versus palatal) of impaction.

However, results of the previous studies carried out with various dissimilar diagnostic records (study casts, ${ }^{7,9,10,13,14,16,18}$, posteroanterior $\mathrm{X}$-rays ${ }^{8}$ and $\mathrm{CBCT}^{5,14,17}$ are in sharp contrast to our results.

Several studies ${ }^{7,10,13,14,16}$ failed to record differences in transverse width between the impaction groups (buccal impaction and palatal impaction) and the control group. So, they stated that, maxillary transverse discrepancy was not considered as a causative factor contributing to maxillary canine impaction, in addition, the presence of impacted canine has no effect on maxillary width.

Al Nimri et al., ${ }^{9}$ found a significantly wider maxillary arch in patients with maxillary canine impaction than those with normal canine eruption. This finding is in contrary to our results may be because they used different measurement method on dental casts.

Sambatro et al., ${ }^{8}$ utilized posteroanterior (PA) cephalograms to report a wider posterior hemi maxilla with larger intermolar and inter premolar widths for patients who later developed maxillary canine impaction.

In contrast to our results, the results of Hong et al., ${ }^{5}$ and Gull et al., ${ }^{17}$ showed similar skeletal and dental transverse maxillary widths in individuals with palatally impacted maxillary canines and others without impaction. Therefore, they concluded that the presence of palatally displaced maxillary canines was not secondary to skeletal or dental maxillary transverse deficiency. Additionally, Ghaffar et al., ${ }^{18}$ revealed no significant difference in the occurrence of maxillary canine impaction in 
mixed dentition patients with and without maxillary transverse discrepancy.

This conflict between results can be explained by the numerous heterogeneity (age, sex, population, inclusion criteria, sample size, methods of measurement) found in other papers.

Results of the present study showed a non-significant difference between the impaction groups and the control group regarding the palatal vault depth. These results agreed with results of Fattahi et al., ${ }^{13}$ and Cacciatore et al., ${ }^{20}$ who found similar palatal vault depth in subjects with an impacted canine whether buccal or palatal and in matched controls.

On the other hand, according to kim et al. ${ }^{15}$ the palatal vault and the intermolar width of patients with palatally impacted canine was deeper and smaller respectively, compared with those with buccal impaction. However, in their study, all variables were measured directly on diagnostic digital model not CBCT images. In addition, they used the relative ratio to evaluate the shape of the palatal because they believed that a relative ratio pretends be more appropriate than an absolute value when evaluating the shape.

In contrast to our study Refaat and El-Desouky ${ }^{14}$ observed that the palatal vault was shallower in females with buccally displaced canine compared with the palatally displaced canine and the control subgroups. They concluded the palatal shape of female subjects is more associated with the presence and position of impacted canines than the male subjects. This difference in the results of the shape of the palatal vault may be attributed to that they investigate male and female subjects separately and not as a combined group.

This study has revealed presence of maxillary transverse discrepancies in subjects with labial and palatal impacted maxillary canines. Therefore, this condition should be considered during diagnosis and treatment plan.

\section{CONCLUSIONS}

Based on the findings of the current study, the following conclusions can be considered:

- The maxillary arch was narrower in subjects with labially or palatally impacted maxillary canines than in subjects with normally erupted maxillary canines.

- There is no statistically significant difference in the maxillary transverse measurements between subjects with labial impacted maxillary canine compared with subjects with palatally impacted maxillary canine.

- Subjects with labial or palatal maxillary canine impaction does not show any statistically significant difference in the palatal vault depth compared with subjects without canine impaction.

\section{REFERENCES}

1. Becker A. The orthodontic treatment of impacted teeth. 2nd ed. London, United Kingdom: Informa Healthcare; 2007.p. 93-174.

2. Bedoya MM, Park JH. A review of the diagnosis and management of impacted maxillary canines. J Am Dent Assoc. 2009;140: 1485-93.

3. Walker L, Enciso R, Mah J. Three-dimensional localization of maxillary canines with cone beam computed tomography. Am J Orthod Dentofac Orthop. 2005; 128:418-23.

4. Alqerban A, Jacobs R, Fieuws S and Willem G. Comparison of two cone beam computed tomographic systems versus panoramic imaging for localization of impacted maxillary canines and detection of root resorption. Eur J Orthod 2011; 33:93-102.

5. Hong W, Radfar R, Chung C. Relationship between the maxillary transverse dimension and palatally displaced canines: a cone beam computed tomographic study. Angle Orthod 2015;85: 440-5.

6. McConnell TL, Hoffman DL, Forbes DP, Janzen EK, Weintraub NH. Maxillary canine impaction in patients with transverse maxillary deficiency. ASDC J Dent Child. 1996; 63: 190-5 
7. Langberg BJ, Peck S. Adequacy of maxillary dental arch width in patients with palatally displaced canines. Am J Orthod Dentofacial Orthop 2000;118: 220-3.

8. Sambataro S, Baccetti T, Franchi L, Antonini F. Early predictive variables for upper canine impaction as derived from posteroanterior cephalograms. Angle Orthod 2005;75: 28-34.

9. Al-Nimri K, Gharaibeh T. Space conditions and dental and occlusal features in patients with palatally impacted maxillary canines: an etiological study. Eur J Orthod 2005; 27: 461-465.

10. Saiar M, Rebellato J, Sheats RD. Palatal displacement of canines and maxillary skeletal width. Am J Orthod Dentofacial Orthop 2006;129: 511-9.

11. Schindel RH, Duffy SL. Maxillary transverse discrepancies and potentially impacted maxillary canines in mixeddentition patients. Angle Orthod 2007;77: 430-5.

12. Ali Rizvil SA, Shaheed M, Ayub A, Shazia Z, Masood O. Association of maxillary transverse discrepancy and impacted maxillary canines. Pakistan Oral Dent J 2012;32: 439-43.

13. Fattahi H, Ghaeed F and Alipour A. Association between maxillary canine impaction and arch dimensions. Aust Orthod J. 2012; 28: 57-62.

14. Refaat W, El-Desouky G. Cone beam computed tomography assessment of the relation between sex and morphology of maxilla in patients with impacted maxillary canine. Egyptian Dental J. 2017; 63: 157-67.

15. Kim Y, Hyun HK, Jang KT. 2. Interrelationship between the position of impacted maxillary canines and the morphology of the maxilla. Am J Orthod Dentofacial Orthop. 2012; 141: 556-62.

16. Yan B, Sun Z, Fields H, Wang L, Luo L. Etiologic factors for buccal and palatal maxillary canine impaction: a perspective based on cone-beam computed tomography analyses. Am J Orthod Dentofacial Orthop 2013; 143:527-34.

17. Gull M, Mushtaq M, Maqbool S. Palatal displacement of maxillary canines and maxillary transverse dimensions. Annals of International Medical and Dental Research 2018; 4: 9-13.
18. Ghaffar F, Sukhia R, Fida M. Association between maxillary transverse discrepancy and occurrence of potentially impacted maxillary canines in mixed dentition patients. International Orthodontics 2019; 17:554-61.

19. Ariza N, Schilling J, Guillén L, Mora G, Cárdenas Y, Castillo A. Maxillary transverse dimensions in subjects with and without impacted canines: A comparative cone-beam computed tomography study. Am J Orthod Dentofacial Orthop 2018; 154:495-503.

20. Cacciatore G, Poletti L, Sforza C. Early diagnosed impacted maxillary canines and the morphology of the maxilla: a three-dimensional study. Progress in Orthodontics 2018; 16: $220-8$.

21. Guillen E, Cárdenas Y, Castillo A, Mora G, Silveira H. Inter-premolar width changes related to the orthodontic traction of maxillary impacted canines in adolescents and young adults: A retrospective CBCT study. International Orthodontics 2020; 18: 480-9.

22. McSherry P, Richardson A. Ectopic eruption of the maxillary canine quantified in three dimensions on cephalometric radiographs between the ages of 5 and 15 years. Eur $\mathbf{J}$ Orthod 1999; 21: 41-8.

23. Botticelli S, Verna C, Paolo M. Cattaneo, Heidmann J and Melsen B. Two- versus three-dimensional imaging in subjects with unerupted maxillary canines. Eur J Orthod 2011; 33: 344-9.

24. Pittayapat P, Willems G, Alqerban A, Coucke W, Rotta F, Souza C, Westphalen F, Jacobs R. Agreement between cone beam computed tomography images and panoramic radiographs for initial orthodontic evaluation. Oral Surg Oral Med Oral Pathol Oral Radiol 2014;117: 111-9.

25. Tadinada A, Mahdian M, Vishwanath M, Allareddy V, Upadhyay M, Yadav S. Evaluation of alveolar bone dimensions in unilateral palatally impacted canine: a conebeam computed tomographic analysis. Eur J Orthod 2015; 37:596-602.

26. Aracena M, Guillen L, Cardenas Y, Mora G. Skeletal and dentoalveolar bilateral dimensions in unilateral palatally impacted canine using cone beam computed tomography. Prog Orthod 2017; 18:7. 